PROCEEDINGS OF THE

AMERICAN MATHEMATICAL SOCIETY

Volume 139, Number 9, September 2011, Pages 3349-3357

S 0002-9939(2011)10739-1

Article electronically published on January 21, 2011

\title{
DEPENDENCE AND ISOLATED EXTENSIONS
}

\author{
VINCENT GUINGONA
}

(Communicated by Julia Knight)

\begin{abstract}
In this paper, we show that if $\varphi(x ; y)$ is a dependent formula, then all $\varphi$-types $p$ have an extension to a $\varphi$-isolated $\varphi$-type, $p^{\prime}$. Moreover, we can choose $p^{\prime}$ to be an elementary $\varphi$-extension of $p$ and $\left|\operatorname{dom}\left(p^{\prime}\right)-\operatorname{dom}(p)\right| \leq$ $2 \cdot \operatorname{ID}(\varphi)$. We show that this characterizes $\varphi$ being dependent. Finally, we give some corollaries of this theorem and draw some parallels to the stable setting.
\end{abstract}

\section{INTRODUCTION}

One major goal of model theory is to classify and characterize certain nice classes of formulas and theories. One such class of formulas that have been explored extensively is the class of stable formulas. More recently, some model theorists have studied a natural generalization of stable formulas known as dependent formulas (also known as NIP formulas for "not the independence property"). There is a characterization of the stability of a formula $\varphi(\bar{x} ; \bar{y})$ in terms of the definability of all $\varphi$-types. A partitioned formula $\varphi(\bar{x} ; \bar{y})$ is stable if and only if all $\varphi$-types are definable by a formula over their domain (see [1]). In this paper, we produce an analogous result for dependent formulas.

The main theorem, Theorem 2.4. characterizes dependent formulas using the related notions of $\varphi$-isolation and $\varphi$-definability. One interesting thing about Theorem 2.4 is that it is a local result; it describes the behavior of a specific $\varphi$-type for a dependent formula $\varphi$ regardless of the complexities of the ambient theory (e.g., even if the whole theory is independent). Another noteworthy fact is that this theorem is a new result even for stable formulas. Consequences to the stable setting are explored in Section 4 .

Our characterization of dependent formulas necessarily differs from the characterization of stable formulas as dependence is a strictly weaker notion. There are two main differences: First, we weaken the requirement that a $\varphi$-type $p$ be definable over $\operatorname{dom}(p)$. Instead, we take a model $M$ containing $\operatorname{dom}(p)$, take an elementary extension $(N ; B)$ of the pair structure $(M ; \operatorname{dom}(p))$, and demand that $p$ be definable over $B$. Second, we strengthen the method by which the $\varphi$-type $p$ is definable. Instead of being merely definable over this expanded set $B$, we demand that there exists an extension of $p$ to a $\varphi$-type $p^{\prime}$ such that $\operatorname{dom}\left(p^{\prime}\right) \subseteq B$ and $p^{\prime}$ is $\varphi$-isolated.

Received by the editors November 6, 2009 and, in revised form, August 16, 2010

2010 Mathematics Subject Classification. Primary 03C45.

Key words and phrases. Definability, dependent, NIP, types.

Special thanks to Chris Laskowski.

The author was partially supported by Laskowski's NSF grants DMS-0600217 and 0901336.

(C)2011 American Mathematical Society

Reverts to public domain 28 years from publication 
This, in turn, implies that $p$ is $\varphi$-definable over $B$. The proof of Theorem 2.4 the isolated extension theorem, is loosely based on a paper by Shelah [2].

In Section 2 we discuss definitions, state the main theorem, and list some consequences of that theorem. The main theorem, Theorem 2.4 is proved in Section 3 , Section 4 is devoted to the stable setting.

\section{Definitions AND THE ISOlAted EXtension THEOREM}

Fix a complete, first-order theory $T$ in a language $L$. For convenience, if $\psi(\bar{x})$ is any formula, then let $\psi(\bar{x})^{0}=\neg \psi(\bar{x})$ and let $\psi(\bar{x})^{1}=\psi(\bar{x})$.

For the first three definitions, fix $\varphi(\bar{x} ; \bar{y})$, a partitioned formula of $L$. By a $\varphi$-type we mean a consistent set of formulas $p(\bar{x})=\left\{\varphi(\bar{x} ; \bar{b})^{s(\bar{b})}: \bar{b} \in B\right\}$ for some set $B$ of elements of the same sort as $\bar{y}$ and some $s \in{ }^{B} 2$ (the set of functions from $B$ to $2=\{0,1\})$. We say that $\operatorname{dom}(p)=B$ and the space of all $\varphi$-types over $B$ is denoted

$$
S_{\varphi}(B)=\{p(x) \text { a } \varphi \text {-type }: \operatorname{dom}(p)=B\} .
$$

For any model $M \models T$, for any $\bar{a}$ from $M$ and any $B$ a set of elements of the same sort as $\bar{y}$ from $M$, let $\operatorname{tp}_{\varphi}(\bar{a} / B)$ be the $\varphi$-type over $B$ given by

$$
\operatorname{tp}_{\varphi}(\bar{a} / B)=\left\{\varphi(\bar{x} ; \bar{b})^{t}: \bar{b} \in B, t<2 \text { such that } M \models \varphi(\bar{a} ; \bar{b})^{t}\right\} .
$$

The above notions can be defined for sets of formulas $\Gamma(\bar{x} ; \bar{y})$ (instead of a single formula) in the obvious way. Throughout this section, when we mention a $\varphi$ type over $B$, look at $\operatorname{tp}_{\varphi}(\bar{a} / B)$, or consider the set $S_{\varphi}(B)$, we want $B$ to be a set of elements of the same sort as $\bar{y}$ (that is, if $\bar{y}=\left(y_{0}, \ldots, y_{n-1}\right)$, then $B$ is a set of $n$-tuples $\bar{b}=\left(b_{0}, \ldots, b_{n-1}\right)$ such that $b_{i}$ is of the same sort as $y_{i}$ for all $\left.i<n\right)$. In Section 3 when we consider $\Delta$-types, we alter this notation slightly for simplification. When we consider the set of formulas $\Delta\left(y ; z_{0}, \ldots, z_{n-1}\right)$ where all the $z_{i}$ 's are of the same sort and $B$ is a set of elements of that sort, we abuse notation and say that a $\Delta$ type is over $B$ when it is actually over $B^{n}$ and we write $\operatorname{tp}_{\Delta}(c / B)$ when we mean $\operatorname{tp}_{\Delta}\left(c / B^{n}\right)$.

Definition 2.1. We say that a set $B$ of elements of the same sort as $\bar{y}$ is $\varphi$ independent if, for all $s \in{ }^{B} 2$, the set of formulas $\left\{\varphi(\bar{x} ; \bar{b})^{s(\bar{b})}: \bar{b} \in B\right\}$ is consistent. We say that $\varphi$ has independence dimension $n<\omega$, denoted $\operatorname{ID}(\varphi)=n$, if $n$ is maximal such that, for some (equivalently any) model $M=T$, there exists a set $B$ of elements of the same sort as $\bar{y}$ from $M$ with $|B|=n$ such that $B$ is $\varphi$-independent. If such an $n$ exists, then we say that $\varphi$ is dependent. If no such $n$ exists, then we say that $\varphi$ is independent.

Notice that when $B$ is finite, $B$ is $\varphi$-independent if and only if $\left|S_{\varphi}(B)\right|=2^{|B|}$.

Definition 2.2. We say that a $\varphi$-type $p(\bar{x})$ is $\varphi$-isolated if there exists a finite $\varphi$-subtype $p_{0}(\bar{x}) \subseteq p(\bar{x})$ such that $p_{0}(\bar{x}) \vdash p(\bar{x})$. We say that a formula $\psi(\bar{x})$ is a $\varphi$-formula if it is of the form $\psi(\bar{x})=\bigwedge_{i<n} \varphi\left(\bar{x} ; \bar{b}_{i}\right)^{s(i)}$ for some $n<\omega$, some elements $\bar{b}_{i}$ of the same sort as $\bar{y}$, and some $s \in{ }^{n} 2$.

We see that a $\varphi$-type $p(\bar{x})$ is $\varphi$-isolated if and only if there exists a $\varphi$-formula $\psi(\bar{x})$ over $\operatorname{dom}(p)$ such that $p(\bar{x})$ is equivalent to $\psi(\bar{x})$. This $\varphi$-formula is simply the conjunction of the finite $\varphi$-subtype $p_{0}(\bar{x})$ given in Definition 2.2 . 
For a model $M \models T$ and a set $B$ of elements of the same sort as $\bar{y}$ from $M$, consider the language $L_{B}=L \cup\left\{P_{B}\right\}$, an expansion of $L$ by adding a single predicate, $P_{B}(\bar{y})$. Let $(M ; B)$ be the obvious $L_{B}$-structure. By " $\left(N ; B^{\prime}\right) \succeq(M ; B)$ " we mean that $\left(N ; B^{\prime}\right)$ is an elementary extension of $(M ; B)$ in the language $L_{B}$.

Definition 2.3. Fix $M \models T$ and a set $B$ of elements of the same sort as $\bar{y}$ from $M$. We say that a $\varphi$-type $p^{\prime}$ is an elementary $\varphi$-extension of the $\varphi$-type $p \in S_{\varphi}(B)$ if $p^{\prime}$ extends $p$ and $\operatorname{dom}\left(p^{\prime}\right) \subseteq B^{\prime}$ for some $\left(N ; B^{\prime}\right) \succeq(M ; B)$.

Now we are ready to state the main theorem of the paper. We give the proof in Section 3 below.

Theorem 2.4 (The isolated extension theorem). For any partitioned formula $\varphi(\bar{x} ; \bar{y})$, the following are equivalent:

(i) $\varphi$ is dependent;

(ii) For all $\varphi$-types $p$, there exists a $\varphi$-isolated elementary $\varphi$-extension of $p$.

Moreover, if the above conditions hold, we can choose $p^{\prime}$ to be a $\varphi$-isolated elementary $\varphi$-extension of $p \in S_{\varphi}(B)$ such that $\left|\operatorname{dom}\left(p^{\prime}\right)-B\right| \leq 2 \cdot \operatorname{ID}(\varphi)$.

We remark on some consequences of the theorem.

Definition 2.5. Fix a partitioned formula $\varphi(\bar{x} ; \bar{y})$, a $\varphi$-type $p(\bar{x})$, and a formula $\psi(\bar{y})$. We say that $\psi$ defines $p$ if, for all $\bar{b} \in \operatorname{dom}(p), \varphi(\bar{x} ; \bar{b}) \in p(\bar{x})$ if and only if $\psi(\bar{b})$ holds. We say that $\psi \varphi$-defines $p$ if it defines $p$ and it is of the form $\psi(\bar{y})=\forall \bar{x}(\gamma(\bar{x}) \rightarrow \varphi(\bar{x} ; \bar{y}))$ for some $\varphi$-formula $\gamma(\bar{x})$.

Merely requiring that a $\varphi$-type has a defining formula has no content. Indeed, for any type $p \in S_{\varphi}(B), p$ is defined by the formula $\varphi(\bar{a} ; \bar{y})$ for any realization $\bar{a}$ of $p$. The strength of having a defining formula is to have one with a controlled domain, preferably over $\operatorname{dom}(p)$. It is known, for example, that for stable formulas $\varphi$, all $\varphi$-types $p$ have a defining formula over $\operatorname{dom}(p)[1$, but when $\operatorname{dom}(p)$ is an arbitrary set, it does not necessarily have a $\varphi$-defining formula over $\operatorname{dom}(p)$.

Notice that if $p$ is $\varphi$-isolated, then $p$ has a $\varphi$-defining formula $\psi$ over $\operatorname{dom}(p)$. Namely, take a $\varphi$-formula $\gamma$ over $\operatorname{dom}(p)$ such that $p(\bar{x})$ is equivalent to $\gamma(\bar{x})$ and let $\psi(\bar{y})=\forall \bar{x}(\gamma(\bar{x}) \rightarrow \varphi(\bar{x} ; \bar{y}))$. It is clear that if $\psi \varphi$-defines $p$, then $\psi$ defines $p$, but the converse does not necessarily hold. We immediately get the following corollary to Theorem 2.4.

Corollary 2.6 (Elementary $\varphi$-definability of types). If $M \models T, \bar{y}$ is a list of variables, and $B$ is a set of elements of the same sort as $\bar{y}$ from $M$, then there exists an elementary extension $\left(N ; B^{\prime}\right) \succeq(M ; B)$ such that, for all dependent formulas $\varphi(\bar{x} ; \bar{y})$, for all $p(\bar{x}) \in S_{\varphi}(B)$, there exists $\psi(\bar{y})$ over $B^{\prime}$ such that $\psi \varphi$-defines $p$.

Proof. Fix $M \models T, B$ from $M$ of the appropriate sort, and $\left(N ; B^{\prime}\right) \succeq(M ; B)$ sufficiently saturated. Then, by Theorem 2.4 there exists $p^{\prime}$, a $\varphi$-isolated elementary $\varphi$-extension of $p$ (with $\operatorname{dom}\left(p^{\prime}\right) \subseteq B^{\prime}$ ). Since $p^{\prime}$ is $\varphi$-isolated, there exists $\psi$ (over $\left.\operatorname{dom}\left(p^{\prime}\right) \subseteq B^{\prime}\right)$ that $\varphi$-defines $p^{\prime}$. Since $p \subseteq p^{\prime}, \psi \varphi$-defines $p$.

Notice that Corollary 2.6 is, on the one hand, stronger than the standard definability of types for stable formulas and, on the other hand, weaker. We get that, for dependent formulas $\varphi, \varphi$-types are not only definable, but $\varphi$-definable. However, the defining formula is not over $\operatorname{dom}(p)$, but over $B^{\prime}$ for some $\left(N ; B^{\prime}\right) \succeq(M ; \operatorname{dom}(p))$.

As in the stable case, this $\varphi$-definability of types leads to a notion of stable embeddability. 
Corollary 2.7 (Elementary stable embeddability). If $M \models T$ for a dependent theory $T, \bar{y}$ is a list of variables, and $B$ is a set of elements of the same sort as $\bar{y}$ from $M$, then there exists an elementary extension $\left(N ; B^{\prime}\right) \succeq(M ; B)$ such that, for all formulas $\varphi(\bar{y})$ over any elementary supermodel of $M$, there exists a formula $\psi(\bar{y})$ over $B^{\prime}$ such that $\varphi(B)=\psi(B)$. Moreover, $\psi(\bar{y})=\forall \bar{x}(\gamma(\bar{x}) \rightarrow \varphi(\bar{x} ; \bar{y}))$ for some $\varphi$-formula $\gamma$.

Proof. Fix $\left(N ; B^{\prime}\right) \succeq(M ; B)$ sufficiently saturated as above. For any fixed formula $\varphi(\bar{y})$, say $\varphi$ is over $N^{\prime} \succeq M$, let $\varphi(\bar{y})=\varphi_{0}(\bar{a} ; \bar{y})$ for $\varphi_{0}(\bar{x} ; \bar{y})$ over $\emptyset$ and $\bar{a}$ from $N^{\prime}$, and let $p(\bar{x})=\operatorname{tp}_{\varphi_{0}}(\bar{a} / B)$. As $\varphi_{0}$ is dependent, by Corollary 2.6. there exists $\psi(\bar{y})$ over $B^{\prime}$ that $\varphi_{0}$-defines $p$. Then, by definition, $\varphi(B)=\psi(B)$.

\section{ThE PRoOF OF THE ISOLATED EXTENSION THEOREM}

To aid notation, assume that the length of $\bar{x}$ and the length of $\bar{y}$ is 1 . Other than having more complicated notation, the general case is identical.

First, to show (ii) implies (i), we exhibit the contrapositive. Assume then that $\varphi(x ; y)$ is independent. By compactness, there exists a model $M$ with an infinite $\varphi$-independent set $B$. Let $\left(N ; B^{\prime}\right) \succeq(M ; B)$. By elementarity, it follows that all finite subsets of $B^{\prime}$ are $\varphi$-independent. Let $p^{\prime}$ be any extension of $p$ to a $\varphi$-type such that $\operatorname{dom}\left(p^{\prime}\right) \subseteq B^{\prime}$. Fix any finite subtype $p_{0}(x) \subseteq p^{\prime}(x)$. Now, for any finite $\varphi$ type $p_{1}(x)$ with $p_{0}(x) \subset p_{1}(x) \subseteq p^{\prime}(x)$, since $\operatorname{dom}\left(p_{1}\right)$ is $\varphi$-independent, we cannot have that $p_{0}(x) \vdash p_{1}(x)$. Thus, $p_{0}(x) \not p^{\prime}(x)$. This shows that no elementary $\varphi$-extension of $p$ is $\varphi$-isolated. Therefore, (ii) implies (i).

To show (i) implies (ii), we first show that the following proposition holds:

Proposition 3.1. For any dependent formula $\varphi(x ; y)$ in a theory $T$, for any model $M \models T$, for any partial type $\Theta(y)$ over $\emptyset$, and for any $B \subseteq \Theta(M)$, there exists $N \succeq M$ and $C \subseteq \Theta(N)$ with $|C| \leq 2 \cdot \operatorname{ID}(\varphi)$ and an extension $p^{\prime}(x) \in S_{\varphi}(B \cup C)$ of $p(x)$ that is $\varphi$-isolated.

Fix $\varphi(x ; y)$ to be a dependent formula in a theory $T$ and $\Theta(y)$ to be any partial type over $\emptyset$. Let $n=\operatorname{ID}(\varphi)$, the independence dimension of $\varphi(x ; y)$. Fix $M \models T, N \succeq M$ sufficiently saturated, $B \subseteq \Theta(M)$, and $p(x) \in S_{\varphi}(B)$. If $B$ is finite, $p$ is already isolated, so assume that $B$ is infinite. Define a set of formulas $\Delta\left(y ; z_{0}, \ldots, z_{n-1}\right)$ as follows:

$$
\Delta\left(y ; z_{0}, \ldots, z_{n-1}\right)=\left\{\exists x\left(\varphi(x ; y)^{t} \wedge \bigwedge_{i<n} \varphi\left(x ; z_{i}\right)^{s(i)}\right): t<2, s \in{ }^{n_{2}}\right\} .
$$

We now define the notion of a good configuration. This allows us to build up the external $C$ in at most $\operatorname{ID}(\varphi)$ steps (adding two elements at a time).

Definition 3.2. A good configuration of $p$ of size $K$ is a sequence $C=\left\{c_{i, t}: i<\right.$ $K, t<2\}$ such that the following conditions hold:

(i) $c_{i, t} \models \Theta(y)$ for all $i<K, t<2$;

(ii) $p(x) \cup\left\{\varphi\left(x ; c_{j, t}\right)^{t}: j<K, t<2\right\}$ is consistent;

(iii) for all $s \in K_{2}$, all $j<K, c_{j, 0}$ and $c_{j, 1}$ have the same $\Delta$-type over $B \cup\left\{c_{i, s(i)}\right.$ : $i \neq j\}$.

If $C$ is a good configuration of $p$ of size $K$, then let $p_{C}(x)=p(x) \cup\left\{\varphi\left(x ; c_{j, t}\right)^{t}\right.$ : $j<K, t<2\}$. 
The first thing to note is that these good configurations are used to extend the type $p$ in a very specific way. These could, a priori, be arbitrarily large. However, the fact that $\varphi$ is dependent forces good configurations to be of bounded size.

Lemma 3.3. If $C=\left\{c_{i, t}: i<K, t<2\right\}$ is a good configuration of $p$ of size $K$, then $K \leq n=\mathrm{ID}(\varphi)$.

Proof. Suppose not; i.e. $K>n$. A good configuration of size $n+1$ explicitly gives us $2^{n+1}$ unique $\varphi$-types over $2(n+1)$ parameters. Using condition (iii), we can translate this into $2^{n+1}$ types over $n+1$ parameters, producing a $\varphi$-independent set of size $n+1$. Specifically, for each $s \in^{n+1} 2$, notice that

$$
\vDash \exists x \bigwedge_{i<n+1} \varphi\left(x ; c_{i, s(i)}\right)^{s(i)}
$$

because $\left\{\varphi\left(x ; c_{i, s(i)}\right)^{s(i)}: i<n+1\right\}$ is a consistent type. Now, notice that for any $j \leq n$

$$
\begin{gathered}
\exists x\left(\bigwedge_{i<j} \varphi\left(x ; c_{i, 0}\right)^{s(i)} \wedge \varphi\left(x ; c_{j, s(j)}\right)^{s(j)} \wedge \bigwedge_{j<i<n+1} \varphi\left(x ; c_{i, s(i)}\right)^{s(i)}\right) \\
\quad \Longrightarrow \exists x\left(\bigwedge_{i<j} \varphi\left(x ; c_{i, 0}\right)^{s(i)} \wedge \varphi\left(x ; c_{j, 0}\right)^{s(j)} \wedge \bigwedge_{j<i<n+1} \varphi\left(x ; c_{i, s(i)}\right)^{s(i)}\right)
\end{gathered}
$$

because $c_{j, 0}$ and $c_{j, 1}$ have the same $\Delta$-type over $\left\{c_{i, 0}: i<j\right\} \cup\left\{c_{i, s(i)}: j<i<\right.$ $n+1\}$. Starting with (3.2), then using (3.3) and induction, we get that:

$$
\models \exists x \bigwedge_{i<n+1} \varphi\left(x ; c_{i, 0}\right)^{s(i)} .
$$

But this holds for any $s \in^{n+1} 2$. This contradicts the fact that $n=\operatorname{ID}(\varphi)$.

If there is a maximal good configuration $C$ for a $\varphi$-type $p$, then we argue in the remaining lemmas that $p_{C}$ is $\varphi$-isolated; if no maximal good configuration exists, then Lemma 3.3 shows that $\varphi$ is not dependent.

Now that we have defined good configurations, we need a sufficient condition for taking a good configuration and building a larger one out of it. Clearly any new $d_{0}$ and $d_{1}$ we would like to add on must realize $\Theta$ and must be so that $\neg \varphi\left(x ; d_{0}\right) \wedge \varphi\left(x ; d_{1}\right)$ is consistent with $p_{C}(x)$. However, the third condition for a good configuration is a bit tricky. Not only do $d_{0}$ and $d_{1}$ have to have the same $\Delta$-type over $B \cup\left\{c_{i, s(i)}: i<K\right\}$, but also each $c_{j, 0}$ and $c_{j, 1}$ have to have the same $\Delta$-type over $B \cup\left\{c_{i, s(i)}: i \neq j\right\} \cup\left\{d_{t}\right\}$. We now give a sufficient condition for being able to add on to good configurations.

Lemma 3.4. If $C=\left\{c_{i, t}: i<K, t<2\right\}$ is a good configuration of $p$ and there exist $d_{0}, d_{1}$ such that:

(i) $d_{0}, d_{1} \models \Theta(y)$,

(ii) $p_{C}(x) \cup\left\{\varphi\left(x ; d_{t}\right)^{t}: t<2\right\}$ is consistent,

(iii) $\operatorname{tp}_{\Delta}\left(d_{0} / B \cup C\right)=\operatorname{tp}_{\Delta}\left(d_{1} / B \cup C\right)$, and

(iv) $\operatorname{tp}_{\Delta}\left(d_{0} / B \cup C\right)$ is finitely satisfiable in $B$,

then $C \cup\left\{d_{0}, d_{1}\right\}$ is a good configuration of $p$ (of size $K+1$ ). 
Proof. Clearly all conditions for $C \cup\left\{d_{0}, d_{1}\right\}$ to be a good configuration of $p$ are met except perhaps the condition that $c_{j, 0}$ and $c_{j, 1}$ have the same $\Delta$-type over $B \cup\left\{c_{i, s(i)}: i \neq j\right\} \cup\left\{d_{t}\right\}$ for all $s \in{ }^{K} 2, t<2$. So suppose this fails, and fix the $s \in{ }^{K} 2$ and $t<2$ where this fails.

Then there exists $\delta$ either an element of $\Delta$ or the negation of an element of $\Delta$ such that $N \models \delta\left(c_{j, 0}, \bar{e}\right) \wedge \neg \delta\left(c_{j, 1}, \bar{e}\right)$ for some $\bar{e}$ from $B \cup\left\{c_{i, s(i)}: i \neq j\right\} \cup\left\{d_{t}\right\}$. Since $c_{j, 0}$ and $c_{j, 1}$ have the same $\Delta$-type over $B \cup\left\{c_{i, s(i)}: i \neq j\right\}$, we must have that $\bar{e}=d_{t} \frown \bar{e}^{\prime}$ for some $\bar{e}^{\prime}$ from $B \cup\left\{c_{i, s(i)}: i \neq j\right\}$. Therefore, we get that

$$
N \models \delta\left(c_{j, 0}, d_{t}, \bar{e}^{\prime}\right) \wedge \neg \delta\left(c_{j, 1}, d_{t}, \bar{e}^{\prime}\right) .
$$

By conditions (iii) and (iv) of the hypothesis, there exists $b \in B$ such that

$$
N \models \delta\left(c_{j, 0}, b, \bar{e}^{\prime}\right) \wedge \neg \delta\left(c_{j, 1}, b, \bar{e}^{\prime}\right) .
$$

But, as $b \frown \bar{e}^{\prime}$ is from $B \cup\left\{c_{i, s(i)}: i \neq j\right\}$, this contradicts the fact that $c_{j, 0}$ and $c_{j, 1}$ have the same $\Delta$-type over $B \cup\left\{c_{i, s(i)}: i \neq j\right\}$.

Fix $C$ to be a maximal good configuration of $p$ so that $p_{C}(x)$ is a $\varphi$-type over $B \cup C$. Let $s(x)$ be any extension of $p_{C}(x)$ to a complete type over $B \cup C$. Define $r_{s}(y)$ as follows:

$$
r_{s}(y)=\left\{\exists x\left(\varphi(x ; y)^{t} \wedge \psi(x)\right): \psi \in s, t<2\right\} \cup \Theta(y) .
$$

Lemma 3.5. $r_{s}$ is not finitely satisfied in $B$.

Proof. Suppose, by means of a contradiction, that $r_{s}$ is finitely satisfied in $B$. Let $\mathcal{D}$ be an ultrafilter on $B$ such that for all $\delta(y) \in r_{s}(y), \delta(B) \in \mathcal{D}$ (this exists by finite satisfiability of $r_{s}$ in $\left.B\right)$. Let $q(y)=\operatorname{Av}(\mathcal{D}, B \cup C)$, the average type of $\mathcal{D}$ over $B \cup C$. That is, for any formula $\delta(y)$ over $B \cup C, \delta(y) \in q(y)$ if and only if $\delta(B) \in \mathcal{D}$. Then $q \in S(B \cup C), q$ extends $r_{s}$, and $q$ is finitely satisfied in $B$. Let $q^{\prime}=\left.q\right|_{\Delta}$

Now notice that $\left\{\exists x\left(\varphi(x ; y)^{t} \wedge \psi(x)\right)\right\} \cup q(y)$ is consistent for each $\psi \in s$ and each $t<2$. Since $s$ is closed under conjunction, by compactness we get that $s(x) \cup$ $\left\{\varphi(x ; y)^{t}\right\} \cup q(y)$ is consistent for each $t<2$. Therefore, $s(x) \cup\left\{\varphi(x ; y)^{t}\right\} \cup q^{\prime}(y) \cup$ $\{\theta(y)\}$ is consistent for each $t<2$ and each $\theta(y)$ a finite conjunction of formulas from $\Theta(y)\left(\right.$ as $\left.q^{\prime}(y) \cup \Theta(y) \subseteq q(y)\right)$. This means that $s(x) \cup\left\{\exists y\left(\varphi(x ; y)^{t} \wedge \theta(y) \wedge \psi(y)\right)\right\}$ is consistent for each $\psi(y)$ a finite conjunction of formulas from $q^{\prime}(y)$ and each $\theta(y)$ a finite conjunction of formulas from $\Theta(y)$. But, since $s$ is a complete type in the $x$ variable, $s$ decides all formulas of the form $\exists y\left(\varphi(x ; y)^{t} \wedge \theta(y) \wedge \psi(y)\right)$. Therefore, we get that

$$
\exists y\left(\varphi(x ; y)^{t} \wedge \theta(y) \wedge \psi(y)\right) \in s(x) .
$$

Choose $\psi_{t}(x)$ to be a finite conjunction of formulas from $q^{\prime}(y)$, and $\theta_{t}(y)$ to be a finite conjunction of formulas from $\Theta(y)$, both for $t<2$. Then $\exists y_{t}\left(\varphi\left(x ; y_{t}\right)^{t} \wedge\right.$ $\left.\theta_{t}\left(y_{t}\right) \wedge \psi_{t}\left(y_{t}\right)\right) \in s(x)$ for $t<2$. Therefore, we get that

is consistent. Now, by compactness,

$$
u\left(x, y_{0}, y_{1}\right)=s(x) \cup\left\{\neg \varphi\left(x ; y_{0}\right) \wedge \varphi\left(x ; y_{1}\right)\right\} \cup q^{\prime}\left(y_{0}\right) \cup q^{\prime}\left(y_{1}\right) \cup \Theta\left(y_{0}\right) \cup \Theta\left(y_{1}\right)
$$

is consistent. So, taking any realization $\left(a, d_{0}, d_{1}\right)$ of $u\left(x, y_{0}, y_{1}\right)$ from $N$, we see that $d_{0}, d_{1} \models \Theta(y), d_{0}, d_{1} \models q^{\prime}(y)$, and $p_{C}(x) \cup\left\{\varphi\left(x ; d_{t}\right)^{t}: t<2\right\}$ is consistent. So conditions (i), (ii), and (iii) of Lemma 3.4 are met. However, since $q$ is finitely 
satisfied in $B, q^{\prime}$ is finitely satisfied in $B$. Therefore, condition (iv) of Lemma 3.4 is met, so $C \cup\left\{d_{0}, d_{1}\right\}$ is a good configuration of $p$. This contradicts the maximality of $C$.

We now show how the non-finite-satisfiability of $r_{s}$ in $B$ leads to a formula definition of $p_{C}(x)$.

Lemma 3.6. For any $C$, a maximal good configuration of $p$, and any $s(x) \in$ $S(B \cup C)$ an extension of $p_{C}(x)$, there exists a formula $\gamma(x) \in s(x)$ such that $\gamma(x) \vdash p_{C}(x)$.

Proof. Consider $r_{s}$ as given above. Then, since $r_{s}$ is not finitely satisfiable in $B$ (and $s$ is closed under conjunctions), there exists $\psi(x) \in s(x)$ such that for all $b \in B, N \models \forall x(\psi(x) \rightarrow \varphi(x ; b))$ or $N \models \forall x(\psi(x) \rightarrow \neg \varphi(x ; b))$ (notice here that $b \models \Theta(y)$ for all $b \in B$, so that the formulas in $\Theta(y) \subseteq r_{s}(y)$ are always realized in $B)$. Let $\gamma(x)$ be defined as follows:

$$
\gamma(x)=\psi(x) \wedge \bigwedge_{i<K, u<2} \varphi\left(x ; c_{i, u}\right)^{u} .
$$

Since $s$ is closed under conjunction and $s$ extends $p_{C}$, we get that $\gamma(x) \in s(x)$. To prove that $\gamma(x) \vdash p_{C}(x)$, notice that for all $b \in B$, there exists $t<2$ such that $\psi(x) \vdash \varphi(x ; b)^{t}$; hence $\varphi(x ; b)^{t} \in s(x)$. But $s$ extends $p_{C}$, so we get that $\varphi(x ; b)^{t} \in p_{C}(x)$. Similarly, $\gamma(x) \vdash \varphi\left(x ; c_{i, u}\right)^{u}$ for all $i<K$ and $u<2$. Therefore, $\gamma(x) \vdash p_{C}(x)$.

Now that we have a formula definition for $p_{C}(x)$ for each $s \in S(B \cup C)$, we see that a single formula is equivalent to $p_{C}(x)$ using compactness. After that, we show that this means that a finite $\varphi$-subtype of $p_{C}(x)$ is equivalent to the whole of $p_{C}(x)$.

Lemma 3.7. If $C=\left\{c_{i, t}: i<K, t<2\right\}$ is a maximal good configuration of $p$, then there exists a formula $\psi(x)$ over $B \cup C$ such that $\psi(x)$ is equivalent to $p_{C}(x)$.

Proof. For each such $s(x) \in S(B \cup C)$ extending $p_{C}(x)$, define $\gamma_{s}(x)$ to be the formula such that $\gamma_{s}(x) \in s(x)$ and $\gamma_{s}(x) \vdash p_{C}(x)$ as given in Lemma 3.6 .

Consider the following partial type over $B \cup C$ :

$$
\Sigma(x)=\left\{\neg \gamma_{s}(x): s \in S(B \cup C) \text { and } s(x) \supseteq p_{C}(x)\right\} \cup p_{C}(x) .
$$

Now $\Sigma(x)$ is inconsistent, since otherwise we would have $a \models p_{C}(x)$, yet $a \not \models$ $\gamma_{s}(x)$ for any $s(x)$ extending $p_{C}(x)$. In particular, $a \not \models \gamma_{s_{0}}(x)$ for $s_{0}=\operatorname{tp}(a / B \cup C)$. This contradicts the fact that $s_{0}(x) \vdash \gamma_{s_{0}}(x)$. Therefore, by compactness, there exists some finite set $S_{0} \subseteq S(B \cup C)$ of types extending $p_{C}$ so that $\Sigma_{0}(x)=$ $\left\{\neg \gamma_{s}(x): s \in S_{0}\right\} \cup p_{C}(x)$ is inconsistent. Let $\psi(x)=\bigvee_{s \in S_{0}} \gamma_{s}(x)$.

Certainly $\psi(x) \vdash p_{C}(x)$ as $\gamma_{s}(x) \vdash p_{C}(x)$ for all $s \in S_{0}$. Conversely, if $a \models p_{C}(x)$, then $a \not \models\left\{\neg \gamma_{s}(x): s \in S_{0}\right\}$ (by the inconsistency of $\Sigma_{0}(x)$ ). Therefore, $a \models \psi(x)$. Hence, $p_{C}(x) \vdash \psi(x)$, as desired.

Lemma 3.8. If $C=\left\{c_{i, t}: i<K, t<2\right\}$ is a maximal good configuration of $p$, then there exists a finite $\varphi$-subtype $p_{0}(x) \subseteq p_{C}(x)$ so that $p_{0}(x) \vdash p_{C}(x)$.

Proof. First let $\psi(x)$ be a formula over $B \cup C$ that is equivalent to $p_{C}(x)$, given by Lemma 3.7. Then consider $\{\neg \psi(x)\} \cup p_{C}(x)$, a partial type over $B \cup C$. This is clearly inconsistent. Therefore, there exists a finite subset $p_{0}(x) \subseteq p_{C}(x)$ such that $\{\neg \psi(x)\} \cup p_{0}(x)$ is inconsistent. That is, $p_{0}(x) \vdash \psi(x)$, and therefore we get that $p_{0}(x) \vdash p_{C}(x)$. 
We are now ready to prove Proposition 3.1 .

Proof of Proposition 3.1. Take $C=\left\{c_{i, t}: i<K, t<2\right\}$ to be any maximal good configuration of $p$. By definition, $C \subseteq \Theta(N)$. By Lemma 3.3, $K \leq n$; hence $|C| \leq 2 \cdot n$. Let $p^{\prime}(x)=p_{C}(x)=p(x) \cup\left\{\varphi\left(x ; c_{i, t}\right)^{t}: i<K, t<2\right\}$. By Lemma 3.8, there exists a finite $p_{0}(x) \subseteq p^{\prime}(x)$ so that $p_{0}(x) \vdash p^{\prime}(x)$. Therefore, $p^{\prime}(x)$ is $\varphi$ isolated.

From here we can conclude that (i) implies (ii) holds for Theorem 2.4

Let $\varphi(x ; y)$ be dependent, fix $M \models T, B \subseteq M$ of elements of the same sort as $y$, and any $\varphi$-type $p \in S_{\varphi}(B)$. Let $\Theta(y)=\left\{P_{B}(y)\right\}$ in the language $L_{B}$ (notice that $\varphi(x ; y)$ is still dependent with the same independence dimension in the theory $\left.T h_{L_{B}}(M ; B)\right)$. Therefore, by Proposition 3.1. there exists $\left(N ; B^{\prime}\right) \succeq(M ; B)$ and $C \subseteq \Theta\left(\left(N ; B^{\prime}\right)\right)=B^{\prime}$ with $|C| \leq 2 \cdot \operatorname{ID}(\varphi)$ and a type $p^{\prime}(x) \in S_{\varphi}(B \cup C)$ extending $p(x)$ such that $p^{\prime}(x)$ is $\varphi$-isolated. Notice then that $p^{\prime}$ is an elementary $\varphi$-extension of $p$ that is $\varphi$-isolated, so condition (ii) holds. Moreover, we get that $\left|\operatorname{dom}\left(p^{\prime}\right)-B\right|=$ $|C| \leq 2 \cdot \mathrm{ID}(\varphi)$, as desired.

Remark 3.9. Finally, we remark that this $C$, hence $p^{\prime}$, depends only on a type over $B$ with enough information to guarantee that $C$ is a good configuration of $p$ of maximal size. For example, take $\bar{c}=\left(c_{i, t}: i<K, t<2\right)$ for $C=\left\{c_{i, t}: i<K, t<2\right\}$ a good configuration of $p$ of maximal size, and let

(i) $q^{\prime}(\bar{y})=\left\{P_{B}\left(y_{i, t}\right): i<K, t<2\right\}$,

(ii) $q^{\prime \prime}(\bar{y})=\left\{\exists x\left(\psi(x) \wedge \bigwedge_{i<K, t<2} \varphi\left(x ; y_{i, t}\right)^{t}\right): \psi(x) \in \bigwedge_{\text {finite }} p(x)\right\}$ (that is, $\psi$ ranges over all finite conjunctions of formulas from $p$ ),

(iii) $q^{\prime \prime \prime}(\bar{y})=\operatorname{tp}_{\Delta}(\bar{c} / B)$, and

(iv) $q(\bar{y})=q^{\prime}(\bar{y}) \cup q^{\prime \prime}(\bar{y}) \cup q^{\prime \prime \prime}(\bar{y})$.

Then, for any $\bar{c}^{\prime}=q(\bar{y})$, the type $p_{\bar{c}^{\prime}}(x)=p(x) \cup\left\{\varphi\left(x ; c_{i, t}^{\prime}\right)^{t}: i<K, t<2\right\}$ is $\varphi$-isolated (and an elementary $\varphi$-extension of $p$ ). Notice here that $q(\bar{y}) \subseteq \operatorname{tp}(\bar{c} / B)$, the complete type of $\bar{c}$ over $B$. Therefore, so long as we choose $\left(N ; B^{\prime}\right) \succeq(M ; B)$ so that $\left(N ; B^{\prime}\right)$ is $|B|^{+}$-saturated, $q(\bar{y})$ is realized in $N$. This allows us to pick $\left(N ; B^{\prime}\right) \succeq(M ; B)$ uniformly so that all $\varphi$-types over $B$ have extensions to $\varphi$-isolated $\varphi$-types with domain contained in $B^{\prime}$.

\section{4. $\varphi$-ISOLATED ELEMENTARY $\varphi$-EXTENSIONS FOR STABLE $\varphi$}

Since stable formulas are, in particular, dependent, all stable formulas have the property of Theorem 2.4 (ii). But what is the $\varphi$-isolated elementary $\varphi$-extension $p^{\prime}(x)$ of a given $\varphi$-type $p(x)$ ? In the interesting case when $p(x)$ is not already $\varphi$ isolated, $p^{\prime}(x)$ is a forking extension of $p(x)$. This follows from the Open Mapping Theorem (i.e., the fact that the restriction map from non-forking $\varphi$-extensions of $S_{\varphi}(A)$ to $S_{\varphi}(A)$ is open) as, if $p$ has a non-forking $\varphi$-isolated extension, then it is already $\varphi$-isolated.

On the issue of uniformity, the results of Theorem 2.4 differ strongly from the standard definability of $\varphi$-types in the stable case. In the case where $\varphi$ is stable, we can use a compactness argument to get a uniform definition of $\varphi$-types. Note, however, that this uniform definition is not necessarily a $\varphi$-definition. In general, one cannot get a uniform $\varphi$-definition of all $\varphi$-types, even in the case where $\varphi$ is stable. 
We use a classic example to illustrate this point. Let $T$ be the theory, in the language $L=\{E\}$ with a single binary relation $E$, stating that $E$ is an equivalence relation with infinitely many $E$-equivalence classes all of infinite size. This theory is certainly stable and even $\aleph_{0}$-stable. Fix $M \models T$ and let $B \subset M$ be a set containing one element from one class, two from another, three from a third class, and so on. Finally, let $\varphi(x ; y, z, w)$ be the formula given by

$$
\varphi(x ; y, z, w)=[(z=w \rightarrow x=y) \wedge(z \neq w \rightarrow E(x, y))]
$$

(so $\varphi$ encodes the two formulas " $x=y$ " and $E(x, y)$ into a single formula). Now let $n \in \omega$ be arbitrary and let $a \in M-B$ be in the $E$-equivalence class with exactly $n$ elements of $B$ in it; call this class $[a]_{E}$. Finally, let $p_{n}(x)=\operatorname{tp}_{\varphi}(a / B)$. Now, for any $\left(N ; B^{\prime}\right) \succeq(M ; B)$, notice that the $E$-equivalence class with exactly $n$ elements from $B$ still has exactly $n$ elements from $B^{\prime}$, so $[a]_{E} \cap B^{\prime}=[a]_{E} \cap B$. However, this shows that any $\varphi$-extension of $p_{n}$ to some $p^{\prime}$ with $\operatorname{dom}\left(p^{\prime}\right) \subseteq B^{\prime}$ is $\varphi$-isolated only by a finite subtype whose domain contains $[a]_{E} \cap B$ (this is because we need the full set $[a]_{E} \cap B$ to say that $x \neq b$ for each $b \in\left([a]_{E} \cap B\right)$ yet $E(x, b)$ for some (equivalently all) $\left.b \in\left([a]_{E} \cap B\right)\right)$. As $\left|[a]_{E} \cap B\right|=n$ and $n<\omega$ was arbitrary, we see from this example that there is no uniform bound on the size of the $\varphi$-isolating $\varphi$-subtype of the elementary $\varphi$-extension given by Theorem 2.4 , even in the stable case.

\section{REFERENCES}

1. Saharon Shelah, Classification theory and the number of nonisomorphic models, NorthHolland Publishing Company, 1978. MR513226 (81a:03030)

2. __ Dependent theories and the generic pair conjecture, preprint, September 2009. Communications in Contemporary Mathematics, submitted.

Department of Mathematics, University of Maryland, College Park, Maryland 20742

E-mail address: vincentg@math.umd.edu

URL: http://www.math.umd.edu/ vincentg/ 